\title{
COVID-19
}

\section{Health Evidence Summary No.109}

\section{Kerry Millington \& Samantha Reddin}

Liverpool School of Tropical Medicine (LSTM) \& Institute of Development Studies

25 January 2021

This weekly COVID-19 health evidence summary (HES) is based on 3.5 hours of desk-based research. The summary is not intended to be a comprehensive summary of available evidence on COVID-19 but aims to make original documents easily accessible to decision makers which, if relevant to them, they should go to before making decisions.

\section{Epidemiology and modelling}

\begin{tabular}{|c|c|c|c|c|}
\hline $\begin{array}{l}\text { Publication } \\
\text { date }\end{array}$ & Title/URL & $\begin{array}{l}\text { Journal/Article } \\
\text { type }\end{array}$ & Summary & Keywords \\
\hline 21.01 .2021 & $\begin{array}{l}\text { Model- } \\
\text { informed } \\
\text { COVID-19 } \\
\text { vaccine } \\
\text { prioritization } \\
\text { strategies by } \\
\text { age and } \\
\text { serostatus }\end{array}$ & $\begin{array}{l}\text { Science | } \\
\text { Article }\end{array}$ & $\begin{array}{l}\text { A mathematical model to } \\
\text { compare five age- } \\
\text { stratified prioritization } \\
\text { strategies } \\
\text { A highly effective } \\
\text { transmission-blocking } \\
\text { vaccine prioritised to } \\
\text { adults ages } 20-49 \text { years } \\
\text { minimised cumulative } \\
\text { incidence but mortality } \\
\text { and years of life lost were } \\
\text { minimised with } \\
\text { prioritisation given to } \\
\text { adults over } 60 y, \text { in most } \\
\text { scenarios } \\
\text { Use of individual-level } \\
\text { serological tests to } \\
\text { redirect doses to } \\
\text { seronegative individuals } \\
\text { improved the impact of } \\
\text { each dose while } \\
\text { potentially reducing } \\
\text { existing inequities in } \\
\text { COVID-19 impact } \\
\text { Whilst maximum impact } \\
\text { prioritisation strategies } \\
\text { were broadly consistent } \\
\text { across countries, this } \\
\text { framework can be used }\end{array}$ & $\begin{array}{l}\text { Vaccine } \\
\text { prioritisation }\end{array}$ \\
\hline
\end{tabular}




\begin{tabular}{|c|c|c|c|c|c|}
\hline & & & & $\begin{array}{l}\text { to compare across } \\
\text { contexts considering } \\
\text { transmission rates, } \\
\text { vaccine rollout speeds, } \\
\text { and estimates of naturally } \\
\text { acquired immunity }\end{array}$ & \\
\hline 20.01 .2021 & $\begin{array}{l}\text { Quarantine } \\
\text { and testing } \\
\text { strategies in } \\
\text { contact } \\
\text { tracing for } \\
\text { SARS-CoV- } \\
2 \text { : a } \\
\text { modelling } \\
\text { study }\end{array}$ & $\begin{array}{l}\text { The Lancet } \\
\text { Public Health } \\
\text { | Article }\end{array}$ & & $\begin{array}{l}\text { A modelling study to } \\
\text { assess the merit of } \\
\text { testing contacts to avert } \\
\text { onward transmission and } \\
\text { to replace or reduce the } \\
\text { length of quarantine for } \\
\text { uninfected contacts } \\
\text { In most countries, } \\
\text { contacts of confirmed } \\
\text { COVID-19 cases are } \\
\text { currently asked to } \\
\text { quarantine for } 14 \text { days } \\
\text { after exposure to limit } \\
\text { onward transmission but } \\
\text { at a social and economic } \\
\text { cost to the individual and } \\
\text { wider society and } \\
\text { potentially reducing } \\
\text { adherence and policy } \\
\text { effectiveness } \\
\text { Findings are that } \\
\text { quarantine until a PCR or } \\
\text { lateral flow antigen (LFA) } \\
\text { test on day } 7 \text { after } \\
\text { exposure (with early } \\
\text { release if negative) might } \\
\text { avert as much } \\
\text { transmission as the 14- } \\
\text { day quarantine period } \\
\text { Also, daily repeated LFA } \\
\text { testing of traced contacts } \\
\text { for } 5 \text { days, with isolation } \\
\text { only after a positive test, } \\
\text { might allow for standard } \\
\text { quarantine to be removed } \\
\text { with a small increase in } \\
\text { transmission risk offset } \\
\text { by increased participation } \\
\text { and adherence to } \\
\text { isolation } \\
\text { Thus testing may allow } \\
\text { for a substantial } \\
\text { reduction in the length of, } \\
\text { or replacement of } \\
\text { quarantine with a small } \\
\text { excess in transmission } \\
\text { risk } \\
\text { Decreasing test and trace } \\
\text { delays and increasing }\end{array}$ & $\begin{array}{l}\text { Quarantine, } \\
\text { testing }\end{array}$ \\
\hline
\end{tabular}




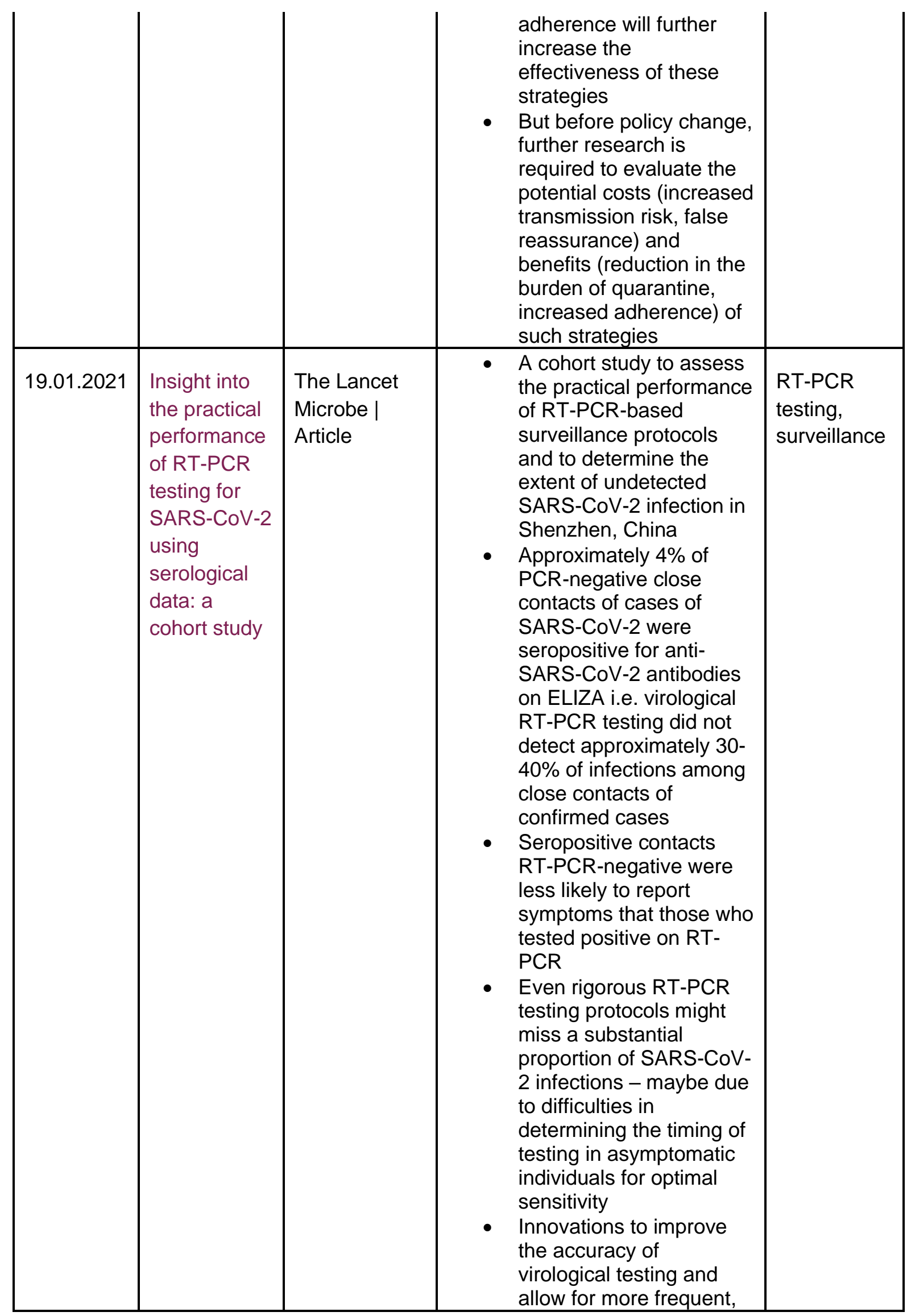




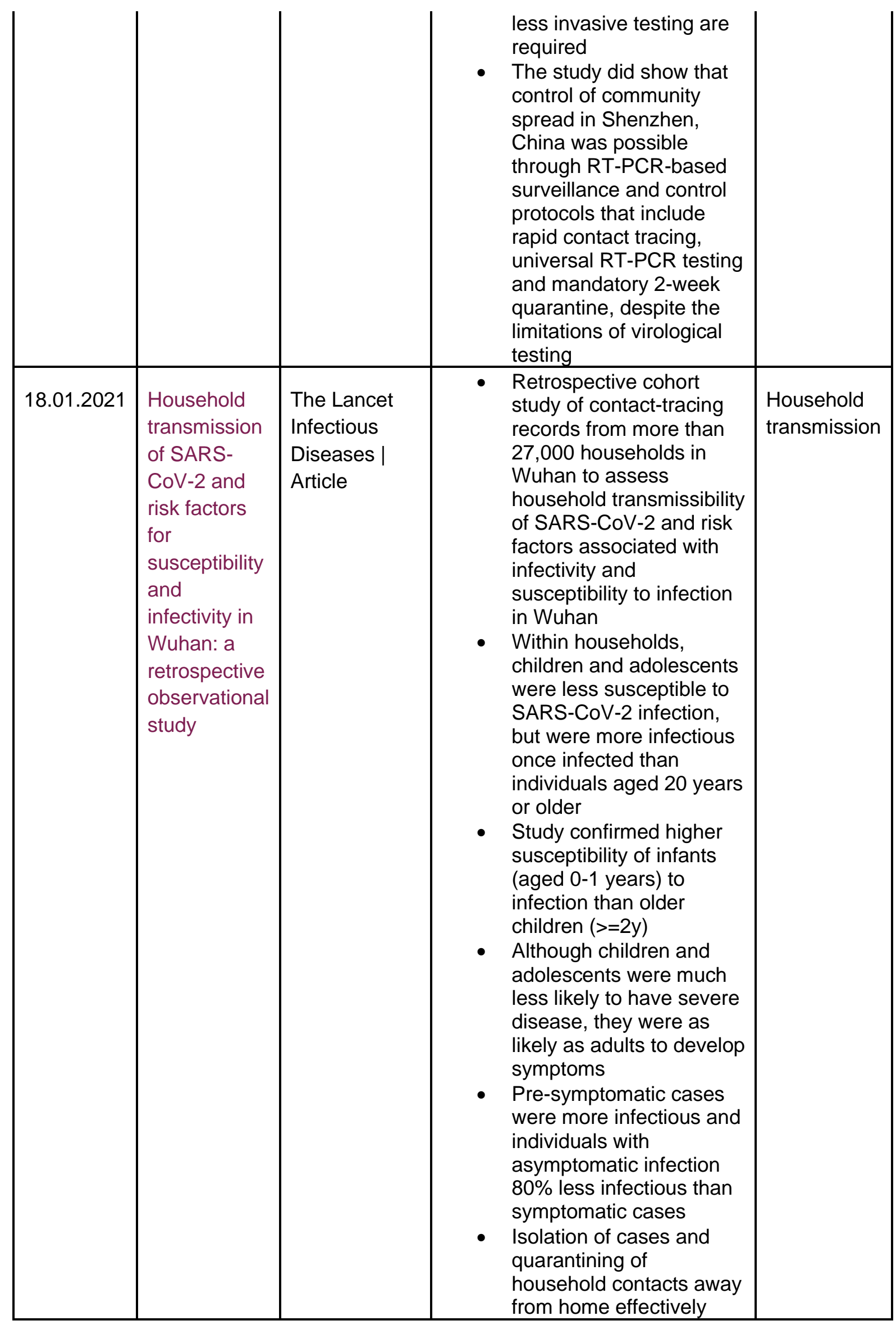




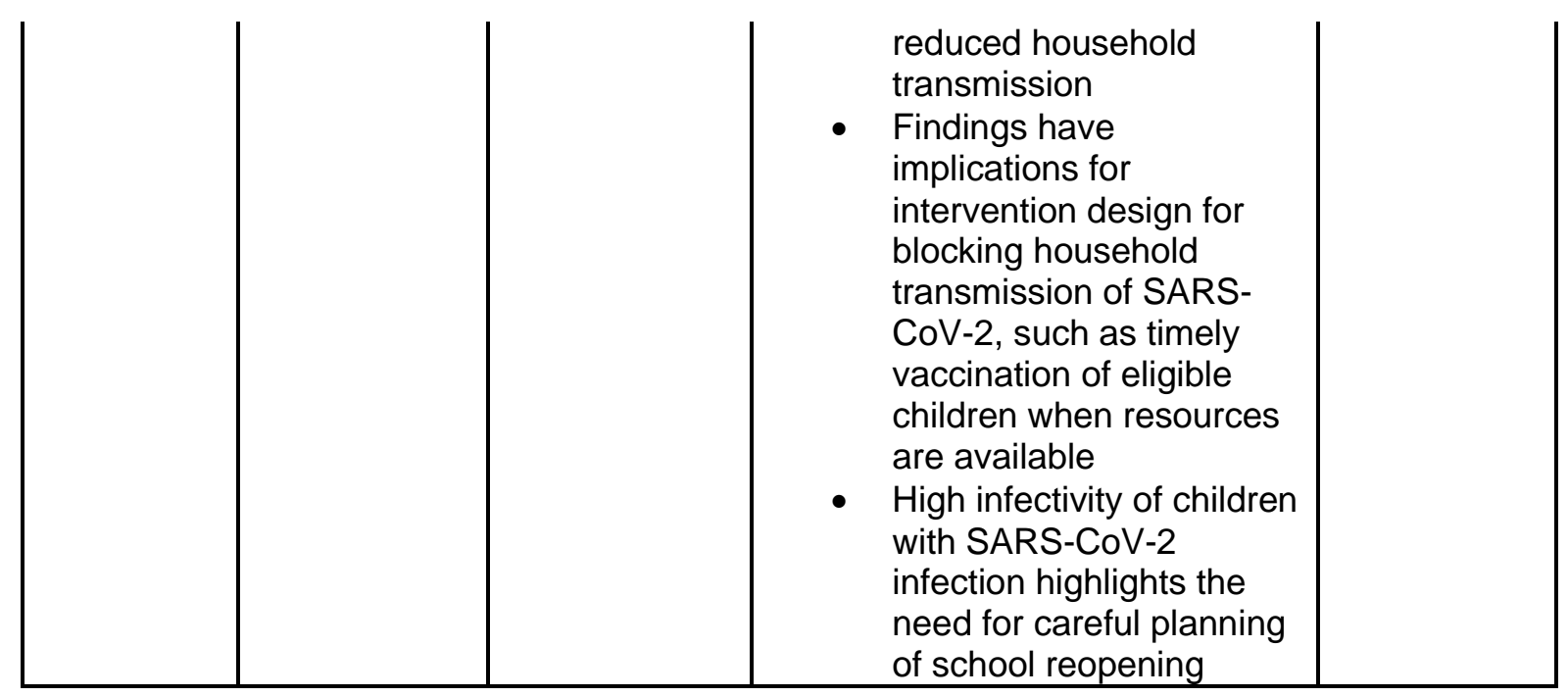

\section{Therapeutics}

\begin{tabular}{|c|c|c|c|c|}
\hline $\begin{array}{l}\text { Publication } \\
\text { date }\end{array}$ & Title/URL & $\begin{array}{l}\text { Journal/Article } \\
\text { type }\end{array}$ & Summary & Keywords \\
\hline 22.01.2021 & $\begin{array}{l}\text { Effect of } \\
\text { anakinra } \\
\text { versus usual } \\
\text { care in adults } \\
\text { in hospital } \\
\text { with COVID- } \\
19 \text { and mild- } \\
\text { to-moderate } \\
\text { pneumonia } \\
\text { (CORIMUNO- } \\
\text { ANA-1): a } \\
\text { randomised } \\
\text { controlled trial }\end{array}$ & $\begin{array}{l}\text { The Lancet } \\
\text { Respiratory } \\
\text { Medicine | } \\
\text { Article }\end{array}$ & $\begin{array}{l}\text { Patients with COVID- } \\
19 \text { pneumonia have an } \\
\text { excess of inflammation } \\
\text { and increased } \\
\text { concentrations of } \\
\text { cytokines including } \\
\text { interleukin (IL-1) } \\
\text { A multicentre, open- } \\
\text { label, Bayesian } \\
\text { randomised clinical trial } \\
\text { to determine whether } \\
\text { anakinra, a } \\
\text { recombinant human IL- } \\
1 \text { receptor antagonist, } \\
\text { could improve } \\
\text { outcomes in patients in } \\
\text { hospital with mild-to- } \\
\text { moderate COVID-19 } \\
\text { pneumonia } \\
153 \text { patients were } \\
\text { screened but } \\
\text { recruitment was } \\
\text { stopped after } 116 \\
\text { patients by the DSMB } \\
\text { Anakinra did not } \\
\text { improve outcomes in } \\
\text { patients with mild-to- } \\
\text { moderate COVID-19 } \\
\text { pneumonia } \\
\text { Efficacy of anakinra } \\
\text { needs to be assessed }\end{array}$ & $\begin{array}{l}\text { Anakinra, IL-1 } \\
\text { receptor } \\
\text { antagonist, } \\
\text { mild-to- } \\
\text { moderate } \\
\text { coVID-19 }\end{array}$ \\
\hline
\end{tabular}




\begin{tabular}{|c|c|c|c|c|c|}
\hline & & & & $\begin{array}{l}\text { in other selected } \\
\text { groups of patients with } \\
\text { more severe COVID-19 }\end{array}$ & \\
\hline 21.01.2021 & $\begin{array}{l}\text { Effect of } \\
\text { bamlanivimab } \\
\text { as } \\
\text { monotherapy } \\
\text { or in } \\
\text { combination } \\
\text { with } \\
\text { etesevimab on } \\
\text { viral load in } \\
\text { patients with } \\
\text { mild to } \\
\text { moderate } \\
\text { COVID-19 }\end{array}$ & $\begin{array}{l}\text { JAMA | } \\
\text { Original } \\
\text { Investigation }\end{array}$ & & $\begin{array}{l}\text { A phase } 2 \text { portion of a } \\
\text { randomised phase } 2 / 3 \\
\text { clinical trial with } 577 \\
\text { patients to assess the } \\
\text { effect of early } \\
\text { treatment with anti- } \\
\text { spike neutralising } \\
\text { antibodies on SARS- } \\
\text { CoV-2 viral load in } \\
\text { outpatients with mild to } \\
\text { moderate COVID-19 } \\
\text { No significant } \\
\text { difference in change in } \\
\text { viral load with } 3 \\
\text { different doses of } \\
\text { bamlanivimab } \\
\text { monotherapy } \\
\text { compared with placebo } \\
\text { Treatment with a } \\
\text { combination of } \\
\text { bamlanivimab and } \\
\text { estesevimab } \\
\text { significantly decreased } \\
\text { SARS-CoV-2 log viral } \\
\text { load at day } 11 \\
\text { compared with placebo }\end{array}$ & $\begin{array}{l}\text { Bamlanivimab, } \\
\text { estesvimab, } \\
\text { mild-to- } \\
\text { moderate } \\
\text { COVID-19 }\end{array}$ \\
\hline
\end{tabular}

\section{Vaccines}

\begin{tabular}{|c|c|c|c|c|}
\hline $\begin{array}{l}\text { Publication } \\
\text { date }\end{array}$ & Title/URL & $\begin{array}{l}\text { Journal/Article } \\
\text { type }\end{array}$ & Summary & Keywords \\
\hline 25.01 .2021 & $\begin{array}{l}\text { mRNA-1273 } \\
\text { vaccine } \\
\text { induces } \\
\text { neutralizing } \\
\text { antibodies } \\
\text { against spike } \\
\text { mutants from } \\
\text { global SARS- } \\
\text { CoV-2 variants }\end{array}$ & $\begin{array}{l}\text { bioRxiv | pre- } \\
\text { print (not peer } \\
\text { reviewed) }\end{array}$ & $\begin{array}{l}\text { - In vitro neutralization } \\
\text { study of sera from } \\
\text { individuals vaccinated } \\
\text { with Moderna mRNA- } \\
1273 \text { vaccine } \\
\text { - } \text { No significant impact on } \\
\text { neutralizing titres against } \\
\text { the B.1.1.7 (UK) variant } \\
\text { relative to prior variants } \\
\text { Six-fold reduction in } \\
\text { neutralizing titres against } \\
\text { the B.1.351 (SA) variant } \\
\text { relative to prior variants, } \\
\text { but remained above } \\
\text { levels that are expected } \\
\text { to be protective } \\
\text { See press release below } \\
\text { for Moderna's plans to }\end{array}$ & $\begin{array}{l}\text { Moderna } \\
\text { vaccine, } \\
\text { variants }\end{array}$ \\
\hline
\end{tabular}




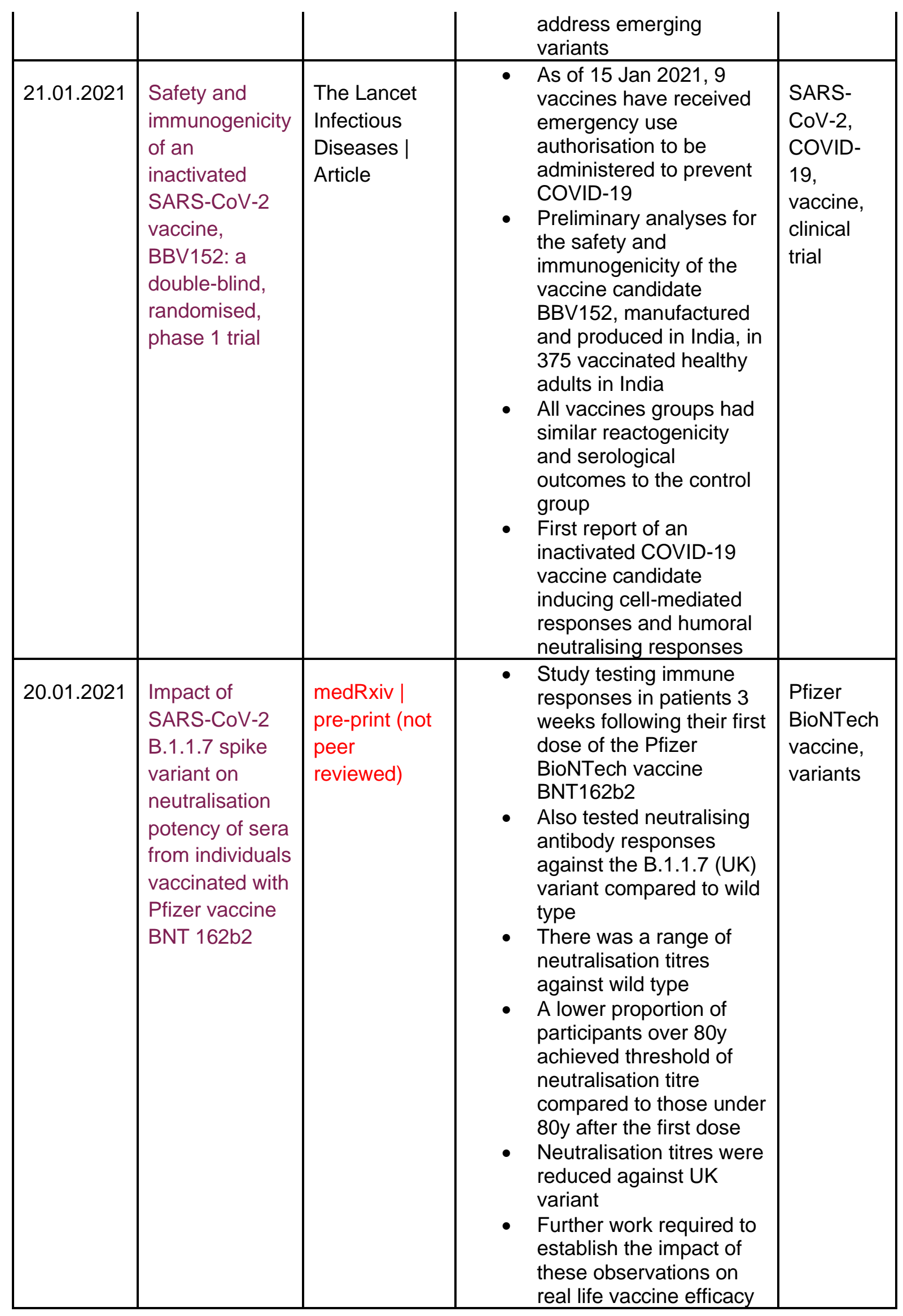




\section{Social Science}

\begin{tabular}{|c|c|c|c|c|}
\hline $\begin{array}{l}\text { Publication } \\
\text { date }\end{array}$ & Title/URL & $\begin{array}{l}\text { Journal/Article } \\
\text { type }\end{array}$ & Summary & Keywords \\
\hline 21.01.2021 & $\begin{array}{l}\text { Navigating } \\
\text { inequities: } \\
\text { a } \\
\text { roadmap } \\
\text { out of the } \\
\text { pandemic }\end{array}$ & $\begin{array}{l}\text { BMJ Global } \\
\text { Health | } \\
\text { Analysis }\end{array}$ & $\begin{array}{l}\text { Presentation of an equity } \\
\text { framework applied to COVID- } \\
19 \text { that sheds light on the full } \\
\text { spectrum of health inequities, } \\
\text { navigates their sources and } \\
\text { intersections, and directs } \\
\text { ethically just interventions } \\
\text { - This equity map guides } \\
\text { surveillance and research to } \\
\text { reveal epidemiological } \\
\text { uncertainties of novel diseases } \\
\text { like COVID-19, recognising } \\
\text { that inequities may exist where } \\
\text { evidence is currently } \\
\text { insufficient } \\
\text { This roadmap can steer global } \\
\text { leadership towards equitable } \\
\text { allocation with diverse } \\
\text { strategies for diverse inequities }\end{array}$ & $\begin{array}{l}\text { Equity } \\
\text { framework }\end{array}$ \\
\hline
\end{tabular}

\section{Comments, Editorials, Opinions, Blogs, News}

\begin{tabular}{|l|l|l|}
\hline $\begin{array}{l}\text { Publication } \\
\text { date }\end{array}$ & Title/URL & Journal | Article type \\
\hline 25.01.2021 & $\begin{array}{l}\text { In a major setback, Merck to stop developing its } \\
\text { two Covid-19 vaccines and focus on therapies }\end{array}$ & Stat news \\
\hline 25.01.2021 & $\begin{array}{l}\text { Moderna COVID-19 vaccine retains neutralizing } \\
\text { activity against emerging variants first identified } \\
\text { in the U.K. and the Republic of South Africa }\end{array}$ & Moderna | Press release \\
\hline 23.01.2021 & $\begin{array}{l}\text { COVID-19: the intersection for education and } \\
\text { health }\end{array}$ & The Lancet | Editorial \\
\hline 22.01.2021 & $\begin{array}{l}\text { Do not repeat mistakes from HIV in COVID-19 } \\
\text { response }\end{array}$ & The Lancet HIV | Editorial \\
\hline
\end{tabular}




\begin{tabular}{|l|l|l|} 
22.01.2021 & $\begin{array}{l}\text { Face masks in the post-COVID-19 era: a silver } \\
\text { lining for the damaged tuberculosis public health } \\
\text { response? }\end{array}$ & $\begin{array}{l}\text { The Lancet Respiratory } \\
\text { Medicine | }\end{array}$ \\
\hline 22.01.2021 & $\begin{array}{l}\text { Full-dose blood thinners decreased need for life } \\
\text { support and improved outcome in hospitalized } \\
\text { COVID-19 patients }\end{array}$ & NIH | News release \\
\hline 21.02.2021 & $\begin{array}{l}\text { Optimism and caution for an inactivated COVID- } \\
19 \text { vaccine }\end{array}$ & $\begin{array}{l}\text { The Lancet Infectious } \\
\text { Diseases | Comment }\end{array}$ \\
\hline 21.01.2021 & Covid: How a £20 gadget could save lives & BBC News \\
\hline 20.01.2021 & $\begin{array}{l}\text { Vaccinating children against Covid-19 - the } \\
\text { lessons of measles }\end{array}$ & NEJM | Perspective \\
\hline 15.01.2021 & Herd immunity by infection is not an option & Science | Perspective \\
\hline 08.01.2021 & SARS-CoV-2 spillover events & Science | Perspective \\
\hline
\end{tabular}




\section{Dashboards \& Trackers}

\begin{tabular}{|c|c|c|c|c|c|c|c|}
\hline $\begin{array}{l}\text { Cases \& deaths: } \\
\text { Global }\end{array}$ & $\begin{array}{l}\text { Cases \& } \\
\text { deaths: } \\
\text { Regional }\end{array}$ & $\begin{array}{l}\text { Cases } \quad \& \\
\text { deaths: } \\
\text { Country }\end{array}$ & $\begin{array}{l}\text { Living evidence \& } \\
\text { policy maps }\end{array}$ & $\begin{array}{l}\text { Current research } \\
\text { including trials }\end{array}$ & Diagnostics & Treatments & Vaccines \\
\hline WHO sitreps & WHO Africa & Ghana & COVID-NMA & WHO & $\begin{array}{l}\text { FIND SARS- } \\
\text { CoV-2 Test } \\
\text { Tracker }\end{array}$ & $\begin{array}{l}\text { Global COVID- } \\
19 \text { Clinical Trial } \\
\text { Tracker }\end{array}$ & CEPI \\
\hline WHO dashboard & $\begin{array}{l}\text { African } \\
\text { Arguments }\end{array}$ & Indonesia & EPPI Centre & $\begin{array}{l}\text { WHO International } \\
\text { Clinical Trials } \\
\text { Registry Platform } \\
\text { (ICTRP) }\end{array}$ & $\begin{array}{l}\text { FIND SARS- } \\
\text { CoV-2 } \\
\text { Diagnostics: } \\
\text { performance } \\
\text { data }\end{array}$ & $\begin{array}{l}\text { US NIH } \\
\text { registered } \\
\text { clinical trials }\end{array}$ & $\begin{array}{l}\text { Vaccine } \\
\text { Centre LSHTM }\end{array}$ \\
\hline $\begin{array}{l}\text { Johns Hopkins } \\
\text { University }\end{array}$ & $\begin{array}{l}\text { European } \\
\text { CDC }\end{array}$ & Nigeria CDC & $\begin{array}{l}\text { Norwegian } \\
\text { Institute of Public } \\
\text { Health }\end{array}$ & Cytel & $\begin{array}{l}\text { Serology-based } \\
\text { tests for COVID- } \\
19\end{array}$ & Solidarity trial & $\begin{array}{l}\text { COVID-19 } \\
\text { Oxford } \\
\text { Vaccine Trial }\end{array}$ \\
\hline WEF & & Sierra Leone & $\begin{array}{l}\text { Oxford C19 } \\
\text { Government } \\
\text { Response } \\
\text { Tracker } \\
\text { (OxCGRT) }\end{array}$ & US NIH & $\begin{array}{l}\text { Our World in } \\
\text { Data: C19 } \\
\text { Testing }\end{array}$ & $\begin{array}{l}\text { COVID-19 } \\
\text { Therapeutics } \\
\text { Accelerator }\end{array}$ & $\begin{array}{l}\text { COVID-19 } \\
\text { Vaccine } \\
\text { Tracker }\end{array}$ \\
\hline
\end{tabular}




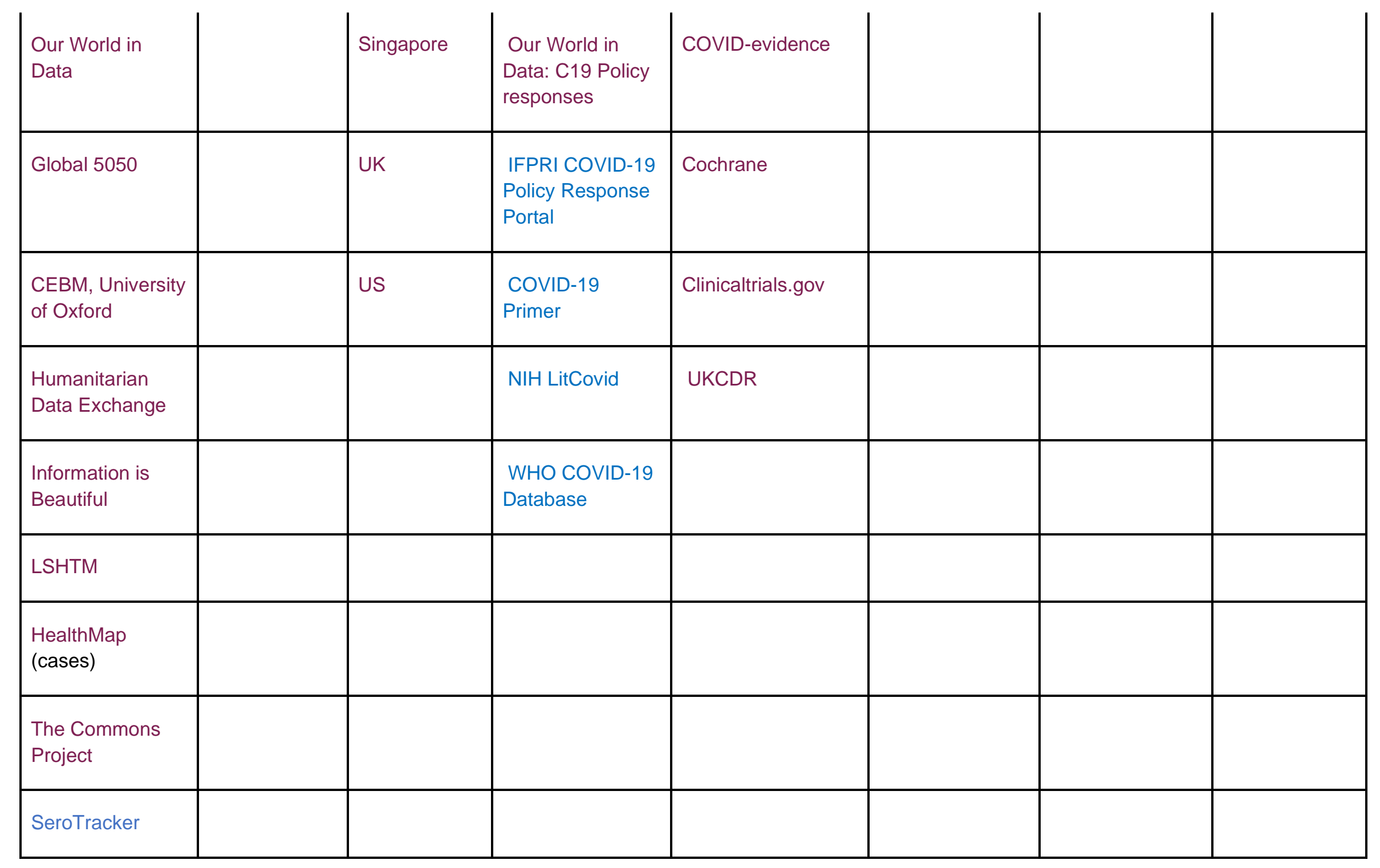




\section{C19 Resource Hubs}

\begin{tabular}{|c|c|c|c|c|c|}
\hline Global & $\begin{array}{l}\text { Regional } \\
\text { \& Country }\end{array}$ & $\begin{array}{l}\text { Academic } \\
\text { journals \& } \\
\text { Publishers }\end{array}$ & $\begin{array}{l}\text { Institutes/Centres/ } \\
\text { Funders/Other }\end{array}$ & $\begin{array}{l}\text { Health } \\
\text { Topics }\end{array}$ & $\begin{array}{l}\text { Social } \\
\text { Sciences }\end{array}$ \\
\hline $\begin{array}{l}\text { WHO } \\
\text { COVID-19 } \\
\text { pandemic }\end{array}$ & $\begin{array}{l}\text { Africa } \\
\text { CDC }\end{array}$ & $\begin{array}{l}\text { Annals of } \\
\text { Internal } \\
\text { Medicine }\end{array}$ & LSTM & $\begin{array}{l}\text { Stop TB } \\
\text { Partnership }\end{array}$ & SSHAP \\
\hline $\begin{array}{l}\text { WHO risk } \\
\text { communicati } \\
\text { on }\end{array}$ & $\begin{array}{l}\text { African } \\
\text { Union }\end{array}$ & BMJ & LSHTM & & IDA \\
\hline WHO Q\&A & $\begin{array}{l}\text { Nigeria } \\
\text { CDC }\end{array}$ & $\begin{array}{l}\text { Bulletin of the } \\
\text { WHO }\end{array}$ & $\begin{array}{l}\text { ICL MRC Centre } \\
\text { for Global } \\
\text { Infectious Disease } \\
\text { Analysis }\end{array}$ & $\begin{array}{l}\text { Global } \\
\text { Menstrual } \\
\text { Collective }\end{array}$ & $\begin{array}{l}\text { Disability and } \\
\text { inclusion }\end{array}$ \\
\hline $\begin{array}{l}\text { WHO Global } \\
\text { research }\end{array}$ & $\begin{array}{l}\text { GeoPoll: } \\
\text { SSA }\end{array}$ & $\begin{array}{l}\text { Cambridge } \\
\text { University } \\
\text { Press }\end{array}$ & ODI & $\begin{array}{l}\text { SLH: } \\
\text { Handwashi } \\
\text { ng in low } \\
\text { resource } \\
\text { settings }\end{array}$ & $\begin{array}{l}\text { Coregroup } \\
\text { IDDC }\end{array}$ \\
\hline $\begin{array}{l}\text { COVID-19 } \\
\text { Solidarity } \\
\text { Response } \\
\text { Fund }\end{array}$ & $\begin{array}{l}\text { Global } \\
\text { Health } \\
\text { Network } \\
\text { Africa }\end{array}$ & Cell Press & $\begin{array}{l}\text { Johns Hopkins } \\
\text { University }\end{array}$ & $\begin{array}{l}\text { RBM } \\
\text { Partnership }\end{array}$ & $\begin{array}{l}\text { Ethics, health } \\
\text { systems \& } \\
\text { COVID-19 }\end{array}$ \\
\hline UN & $\begin{array}{l}\text { African } \\
\text { Academy } \\
\text { of } \\
\text { Sciences }\end{array}$ & Cochrane & $\begin{array}{l}\text { Center for Global } \\
\text { Development }\end{array}$ & $\begin{array}{l}\text { Epidemic } \\
\text { Preparedne } \\
\text { ss } \\
\text { Innovations }\end{array}$ & \begin{tabular}{|l|} 
Social \\
Development \\
Direct C19 \\
blog series
\end{tabular} \\
\hline UN Women & $\begin{array}{l}\text { Africa } \\
\text { Evidence } \\
\text { Network }\end{array}$ & Elsevier & CMMID Repository & & \\
\hline UNOCHA & $\begin{array}{l}\mathrm{OCHA} \\
\text { Southern } \\
\text { and }\end{array}$ & $\begin{array}{l}\text { Health Policy } \\
\text { and Planning }\end{array}$ & $\begin{array}{l}\text { Norwegian Institute } \\
\text { of Public Health }\end{array}$ & & \\
\hline
\end{tabular}




\begin{tabular}{|c|c|c|c|c|}
\hline & $\begin{array}{l}\text { Eastern } \\
\text { Africa } \\
\text { COVID-19 } \\
\text { Digest }\end{array}$ & & & 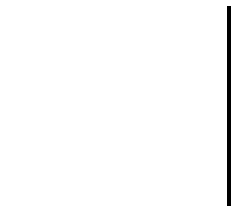 \\
\hline UNHCR & $\begin{array}{l}\text { South } \\
\text { African } \\
\text { Governme } \\
\text { nt }\end{array}$ & JAMA Network & $\begin{array}{l}\text { Oxford Centre for } \\
\text { Evidence-based } \\
\text { Medicine }\end{array}$ & \\
\hline UNICEF & & The Lancet & HEART & \\
\hline UNESCO & & $\begin{array}{l}\text { medRxiv and } \\
\text { bioRxiv } \\
\text { (Preprints) }\end{array}$ & UKRI & \\
\hline UN WFP & & NEJM & Evidence Aid & \\
\hline GOARN & & $\begin{array}{l}\text { Oxford } \\
\text { University } \\
\text { Press }\end{array}$ & $\mathrm{NIH}$ & \\
\hline EPI-WIN & & PLoS & $\begin{array}{l}\text { IFPRI Resources } \\
\text { and Analyses of } \\
\text { C19 Impact }\end{array}$ & \\
\hline World Bank & & SAGE journals & $\begin{array}{l}\text { Prevent } \\
\text { Epidemics }\end{array}$ & \\
\hline $\begin{array}{l}\text { Our World in } \\
\text { Data }\end{array}$ & & Science & & \\
\hline $\begin{array}{l}\text { COVID-19 } \\
\text { Narratives by } \\
\text { David } \\
\text { Nabarro }\end{array}$ & & Springer Nature & & \\
\hline Reliefweb & & $\begin{array}{l}\text { SSRN } \\
\text { (Preprints) }\end{array}$ & & \\
\hline
\end{tabular}




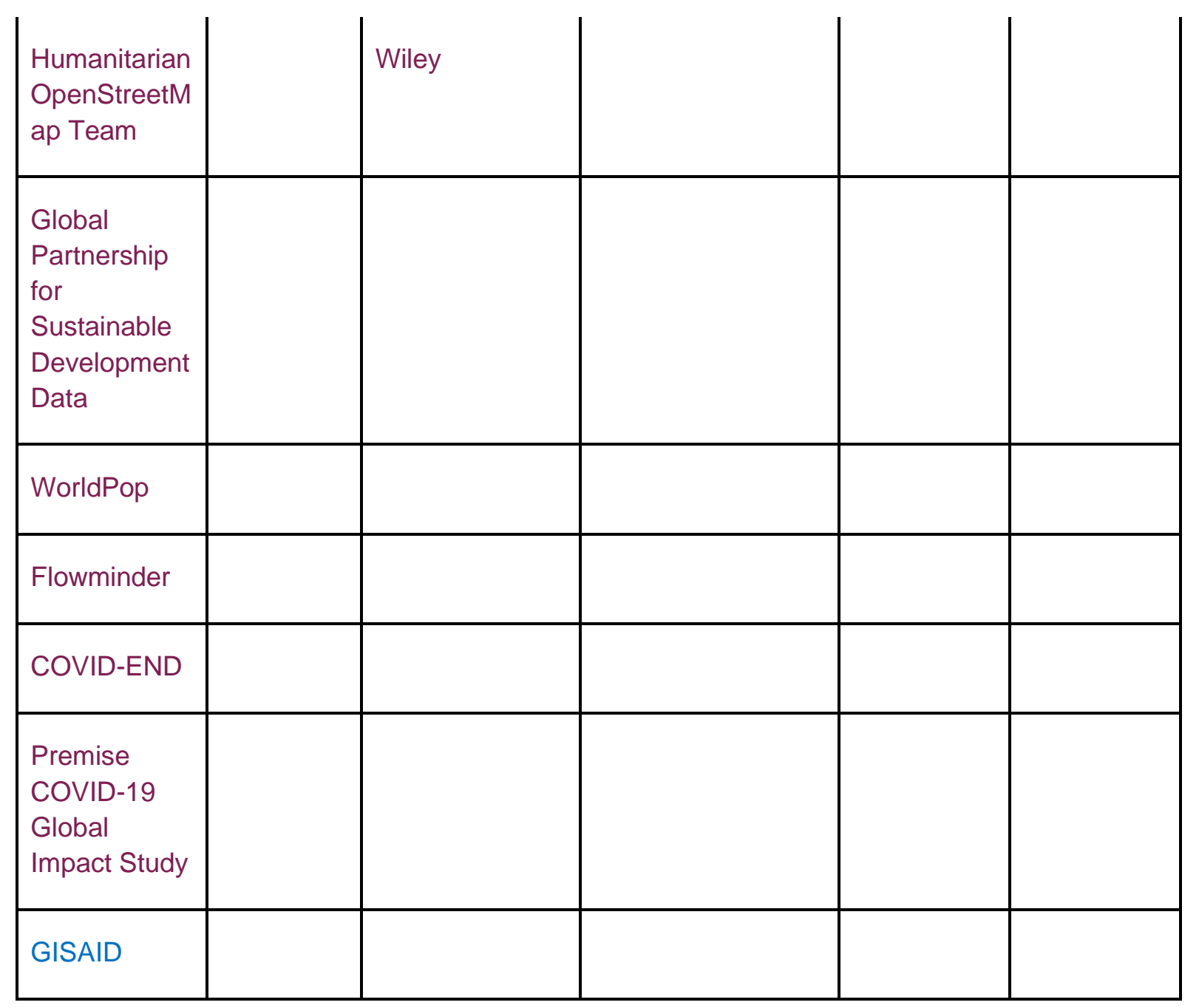

\section{Online learning \& events}

\begin{tabular}{|l|l|l|l|l|}
\hline Date & Title/URL & $\begin{array}{l}\text { Online } \\
\text { learning/event }\end{array}$ & Duration & Lead \\
\hline 14.01 .2021 & $\begin{array}{l}\text { Evidence to impact in } \\
\text { crisis: how have we } \\
\text { measured up during } \\
\text { the COVID-19 } \\
\text { pandemic? }\end{array}$ & Webinar & 1 h 30 & CGD \\
\hline 04.12 .2020 & $\begin{array}{l}\text { CoVID-19, supply } \\
\text { chain resilience and } \\
\text { global trade }\end{array}$ & Webinar & $1 \mathrm{~h}$ & CGD \\
\hline 03.12.2020 & $\begin{array}{l}\text { More money for } \\
\text { health services: What }\end{array}$ & $\begin{array}{l}\text { WHO \& CGD } \\
\text { Health systems }\end{array}$ & 1 h 30 & Joe Kutzin \\
\hline
\end{tabular}




\begin{tabular}{|c|c|c|c|c|}
\hline & $\begin{array}{l}\text { is the tole of PFM in } \\
\text { the "new normal"? }\end{array}$ & $\begin{array}{l}\text { Governance \& } \\
\text { Financing }\end{array}$ & & \\
\hline 01.12 .2020 & $\begin{array}{l}\text { Solutions and support } \\
\text { for the mental } \\
\text { wellbeing of } \\
\text { community health } \\
\text { workers on the } \\
\text { COVID-19 frontline }\end{array}$ & Webinar & & $\begin{array}{l}\text { HSG TWG on CHWs } \\
\text { with The George } \\
\text { Institute for Global } \\
\text { Health }\end{array}$ \\
\hline 19.11 .2020 & $\begin{array}{l}\text { Looking at the } \\
\text { pandemic with a } \\
\text { gender lens }\end{array}$ & $\begin{array}{l}\text { Live Twitter } \\
\text { conversation }\end{array}$ & & SSHAP \\
\hline 16.11 .2020 & $\begin{array}{l}\text { HIFA and WHO } \\
\text { collaborate to } \\
\text { promote sharing of } \\
\text { experience and } \\
\text { expertise around the } \\
\text { maintenance of } \\
\text { essential health } \\
\text { services during (and } \\
\text { after) the pandemic }\end{array}$ & $\begin{array}{l}\text { 4-week } \\
\text { discussion } \\
\text { starting } 16 \text { Nov }\end{array}$ & & HIFA \\
\hline 10.11 .2020 & $\begin{array}{l}\text { COVID-19 vaccine } \\
\text { predictions part 2: } \\
\text { estimating the time } \\
\text { before we approve } \\
\text { efficacious COVID-19 } \\
\text { vaccines }\end{array}$ & Online event & $1 \mathrm{~h} 30$ & CGD \\
\hline 16.10 .2020 & $\begin{array}{l}\text { Financing a Global } \\
\text { Public Health } \\
\text { Response }\end{array}$ & Online event & $1 \mathrm{~h} 30$ & CGD \\
\hline 02.10 .2020 & $\begin{array}{l}\text { Understanding and } \\
\text { Improving COVID-19 } \\
\text { Vaccine Portfolio }\end{array}$ & Online event & $1 \mathrm{~h} 30$ & CGD \\
\hline 21.09 .2020 & $\begin{array}{l}\text { Mitigating the } \\
\text { Economic and Health } \\
\text { Impact of COVID-19 } \\
\text { across Africa }\end{array}$ & Online event & $1 \mathrm{~h} 30$ & CGD, GF, AU \\
\hline
\end{tabular}




\begin{tabular}{|c|c|c|c|c|}
\hline June 2020 & $\begin{array}{l}\text { OpenWHO, the free, } \\
\text { open-access learning } \\
\text { platform for health } \\
\text { emergencies, now } \\
\text { offers } 10 \text { online } \\
\text { courses related to } \\
\text { COVID19. }\end{array}$ & Online courses & Varies & WHO \\
\hline $\begin{array}{l}\text { Available } \\
\text { now }\end{array}$ & $\begin{array}{l}\text { Standard } \\
\text { precautions: } \\
\text { Environmental } \\
\text { cleaning and } \\
\text { disinfection }\end{array}$ & Online course & 1 hour & WHO \\
\hline $\begin{array}{l}\text { Available } \\
\text { now }\end{array}$ & $\begin{array}{l}\text { COVID-19: Effective } \\
\text { Nursing in Times of } \\
\text { Crisis }\end{array}$ & Online course & $\begin{array}{l}2 \text { weeks - } \\
2 \text { hours } \\
\text { per week }\end{array}$ & $\begin{array}{l}\text { Johns Hopkins School } \\
\text { of Nursing }\end{array}$ \\
\hline $\begin{array}{l}\text { Available } \\
\text { now }\end{array}$ & $\begin{array}{l}\text { WHO Academy and } \\
\text { WHO Info mobile } \\
\text { applications }\end{array}$ & Mobile app & & WHO \\
\hline $\begin{array}{l}\text { Available } \\
\text { now }\end{array}$ & $\begin{array}{l}\text { COVID-19: } \\
\text { Pandemics, } \\
\text { Modelling and Policy }\end{array}$ & Online learning & $\begin{array}{l}2 \text { weeks | } \\
2 \text { hours } \\
\text { weekly } \\
\text { study }\end{array}$ & $\begin{array}{l}\text { FutureLearn UNESCO } \\
\text { UNITWIN Complex } \\
\text { Systems Digital } \\
\text { Campus/Open } \\
\text { University }\end{array}$ \\
\hline 11.5 .2020 & $\begin{array}{l}\text { COVID-19 Contact } \\
\text { Tracing course }\end{array}$ & Online learning & 5 hours & $\begin{array}{l}\text { Johns Hopkins } \\
\text { Bloomberg School of } \\
\text { Health }\end{array}$ \\
\hline $\begin{array}{l}7-28 \text { May } \\
2020\end{array}$ & $\begin{array}{l}\text { Virtual Evidence } \\
\text { Weeks }\end{array}$ & 5 sessions & $1 \mathrm{~h} 30$ & $\begin{array}{l}\text { International Initiative } \\
\text { for Impact Evaluation } \\
(3 i e)\end{array}$ \\
\hline $\begin{array}{l}\text { Tuesdays } \\
\text { at } 1700 \\
\text { CEST } \\
\text { (Geneva } \\
\text { time) \& } \\
\text { Thursdays } \\
\text { 0830 CEST }\end{array}$ & $\begin{array}{l}\text { COVID-19 Open } \\
\text { online brief with Dr } \\
\text { David Nabarro }\end{array}$ & Event & $1 \mathrm{~h}$ & $4 S D$ \\
\hline
\end{tabular}




\begin{tabular}{|c|c|c|c|c|}
\hline $\begin{array}{l}\text { (Geneva } \\
\text { time) }\end{array}$ & & & & \\
\hline $\begin{array}{l}\text { Available } \\
\text { now }\end{array}$ & $\begin{array}{l}\text { Emerging respiratory } \\
\text { viruses, including } \\
\text { COVID-19: methods } \\
\text { for detection, } \\
\text { prevention, response } \\
\text { and control }\end{array}$ & Online learning & 3 hours & WHO \\
\hline $\begin{array}{l}\text { Available } \\
\text { now }\end{array}$ & $\begin{array}{l}\text { Responding to } \\
\text { COVID-19: Real-time } \\
\text { training for the } \\
\text { coronavirus disease } \\
\text { outbreak }\end{array}$ & Online learning & $\begin{array}{l}\text { Multiple } \\
\text { self-paced } \\
\text { course }\end{array}$ & WHO \\
\hline $\begin{array}{l}25 \text { May } \\
2020\end{array}$ & $\begin{array}{l}\text { COVID-19: Tackling } \\
\text { the Novel } \\
\text { Coronavirus }\end{array}$ & Online learning & $\begin{array}{l}3 \text { weeks | } \\
4 \text { hours } \\
\text { weekly } \\
\text { study }\end{array}$ & $\begin{array}{l}\text { FutureLearn } \\
\text { LSHTM/UK PHRST }\end{array}$ \\
\hline $\begin{array}{l}\text { Available } \\
\text { online now } \\
\text { without } \\
\text { mentors. } \\
\text { Updated } \\
\text { version will } \\
\text { commence } \\
\text { early June } \\
2020\end{array}$ & $\begin{array}{l}\text { COVID-19 } \\
\text { Diagnostics and } \\
\text { Testing }\end{array}$ & Online learning & $\begin{array}{l}3 \text { weeks | } \\
3 \text { hours } \\
\text { weekly } \\
\text { study }\end{array}$ & $\begin{array}{l}\text { FutureLearn } \\
\text { FIND/LSHTM/ASLM }\end{array}$ \\
\hline 6 April 2020 & $\begin{array}{l}\text { COVID-19 Critical } \\
\text { Care: Understanding } \\
\text { and Application }\end{array}$ & Online learning & $\begin{array}{l}5 \text { weeks | } \\
1 \text { hour } \\
\text { weekly } \\
\text { study }\end{array}$ & $\begin{array}{l}\text { FutureLearn University } \\
\text { of Edinburgh \& Royal } \\
\text { College of Physicians } \\
\text { of Edinburgh }\end{array}$ \\
\hline $\begin{array}{l}\text { Available } \\
\text { now }\end{array}$ & $\begin{array}{l}\text { COVID-19 supporting } \\
\text { online courses }\end{array}$ & Online learning & $\begin{array}{l}\text { Multiple } \\
\text { self-paced } \\
\text { course }\end{array}$ & BMJ Learning \\
\hline
\end{tabular}




\section{Suggested citation}

Millington, K.A. and Reddin, S. (2021). COVID-19 Health Evidence Summary No.109. K4D

Evidence Summary. Brighton, UK: Institute of Development Studies. DOI:

10.19088/K4D.2021.012

\section{Rapid review methodology}

The rapid weekly search for peer-reviewed literature is carried out through a PubMed search with the following keywords ("COVID-19" OR "severe acute respiratory syndrome coronavirus 2" OR "2019-nCoV" OR "SARS-CoV2" OR "2019nCoV" OR "coronavirus" ) AND ("Africa" OR "South Asia" OR "Developing" OR "low-income" OR "low income" OR "lower-middle income" OR "low and middle income" OR "LMIC" OR "LIC" OR "global south") OR ("poverty") OR ("equity" OR "equities"), restricted to articles published in the previous 2 to 3 days, in English. This is complemented by a search of the homepage of the following high-impact global health journals: The Lancet journals, New England Journal of Medicine, Nature, JAMA, Annals of Internal Medicine, Cochrane Reviews, BMJ Global Health, the PLoS journals and a Twitter search of their Twitter pages. A search also of preprints from bioRxiv and medRxiv. Please note that papers that have not been peer-reviewed are highlighted in red. All primary research papers that relate to the primary and secondary impacts of the COVID-19 response in LMICs, and disease control and health system responses are included. Articles related to tackling the secondary impacts on other sectors are not included. Additional commentaries, opinions, and commissioned pieces are selected based on relevance.

The search for dashboards, guidelines, tools, editorials, comments, blogs, opinions and news is through the academic journals listed above, C19 resource hubs and following lead academics and professionals on Twitter.

\section{About this report}

This weekly COVID-19 health evidence summary (HES) is based on 3.5 hours of desk-based research. The summary is not intended to be a comprehensive summary of available evidence on COVID-19 but aims to make original documents easily accessible to decision makers which, if relevant to them, they should go to before making decisions. The HES are not intended to replace medical or professional advice and the researcher or the K4D consortium cannot be held responsible for any decisions made about COVID-19 on the basis of the HES alone. K4D services are provided by a consortium of leading organisations working in international development, led by the Institute of Development Studies (IDS), with Education Development Trust, Itad, University of Leeds Nuffield Centre for International Health and Development, Liverpool School of Tropical Medicine (LSTM), University of Birmingham International Development Department (IDD) and the University of Manchester Humanitarian and Conflict Response Institute (HCRI).

This evidence summary was prepared for the UK Government's Foreign, Commonwealth and Development Office (FCDO) and its partners in support of pro-poor programmes. Except where otherwise stated, it is licensed for non-commercial purposes under the terms of the Open Government Licence v3.0. K4D cannot be held responsible for errors, omissions or any consequences arising from the use of information contained in this health evidence summary. Any views and opinions expressed do not necessarily reflect those of FCDO, K4D or any other contributing organisation.

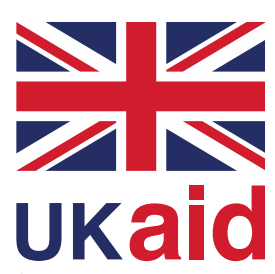

from the British people

(C) Crown copyright 2021 